\title{
Papilomavírus humano como fator etiológico do carcinoma epidermoide bucal
}

\author{
Human papillomavirus as an etiological factor of epidermoid \\ carcinoma
}

\author{
1 Amanda Andressa de Souza Carvalho carvalho.amandasouza@gmail.com \\ 2 Graziele de Souza Diniz \\ 2 Lúbia Cerqueira Costa \\ 2 Stéphany Souza de Araújo \\ 3 Prof. Dr. Josemar Parreira Guimarães
}

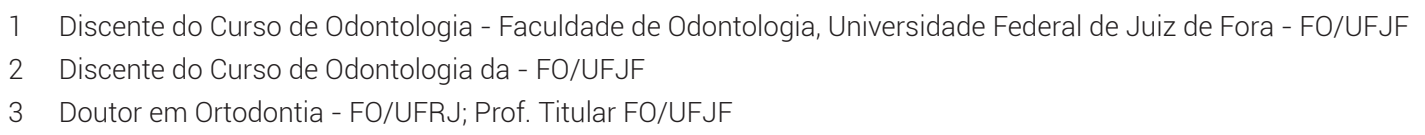

\section{Resumo}

O Papilomavírus Humano (HPV) dos tipos 16 e 18 possui elevada capacidade carcinogênica, estando associado a diversos tumores malignos. Pesquisas indicam que o número de indivíduos que contraem HPV está aumentando de forma alarmante nos últimos anos, o que faz essa infecção apresentar um potencial endêmico. Nessa perspectiva, este estudo aborda alguns aspectos moleculares, histopatológicos, qualitativos e clínicos que envolvem o carcinoma epidermoide bucal (CEB) e sua relação com o HPV, tendo como objetivo primário elucidar os fatores que a literatura pesquisada utiliza para demonstrar a relação existente entre ambos. Já como objetivo secundário, aponta-se a necessidade de enfatizar a importância da atuação do cirurgião-dentista na detecção precoce de tais patologias e na disseminação do conhecimento sobre tal temática. Para tal, foi realizada uma revisão de literatura, utilizando-se as bases de dados SCIELO, LILACS (BVS), PUBMED/MEDLINE, REVODONTO e Portal CAPES, buscando estudos que foram publicados no período de 2014 a 2019.

\section{Palavras-chave}

Câncer bucal. Carcinoma epidermoide. HPV-16. HPV-18. Papilomavírus humano (HPV).

\begin{abstract}
The Human Papillomavirus (HPV) types 16 and 18 have a high carcinogenic capacity, being associated with several malignant tumors. Research indicates that the number of individuals who contract HPV is increasing at an alarming rate in recent years, which makes this infection present an endemic potential. Based on this, this study addresses some molecular, histopathological, qualitative and clinical aspects that involve oral squamous cell carcinoma (CEB) and its relationship with $H P V$, with the primary objective of elucidating the factors that the researched literature points to demonstrate the existing relationship between both. As a secondary objective, there is a need to emphasize the importance of the performance of the Dental Surgeon in the early detection of such pathologies and in the dissemination of knowledge on such subject. To this end, a literature review was carried out in the SCIELO, LILACS (BVS), PUBMED / MEDLINE, REVODONTO and CAPES Portal databases, looking for studies that were published from 2014 to 2019.
\end{abstract}

\section{Keywords}

Oral cancer. Squamous cell carcinoma. HPV-16. HPV-18. Human papillomavirus (HPV).

\section{Como você deve citar?}

CARVALHO, Amanda Andressa de Souza et al. Papilomavírus humano como fator etiológico do carcinoma epidermoide bucal. Cadernos UniFOA, Volta Redonda, n. 43, p. 159-167, agosto 2020. 


\section{INTRODUÇÃO}

De acordo com o Instituto Nacional do Câncer (INCA)', os carcinomas são definidos como alterações de potenciais malignos que provocam o crescimento desordenado de células presentes na pele ou nas mucosas.

Sabe-se que o carcinoma epidermoide é uma das alterações malignas neoplásicas mais frequentes na área de cabeça e pescoço ${ }^{2-3}$, sendo responsável por 355.000 óbitos no mundo4.

Segundo a literatura, o Papilomavírus Humano (HPV) está adquirindo um caráter endêmico, o que é explicado pelo elevado número de indivíduos que praticam relações sexuais desprotegidas, atitude essa que é caracterizada como uma forma de transmissão do vírus ${ }^{2-14-5}$.

A correlação da infecção pelo HPV com a etiologia do câncer bucal foi inicialmente indicada por Syrjanen et al. ${ }^{6}$, em 1983, quando associou-se as modificações celulares encontradas em lesões malignas da boca com alterações encontradas no câncer de colo uterino.

No decorrer dos anos, diversas outras pesquisas foram sendo publicadas com o objetivo de elucidar essa relação apontada pelo autor supracitado. Notou-se, então, que tais estudos convergiam e apontavam para o fato de que o HPV-16 e o HPV-18 estão mais estreitamente relacionados com os tumores malignos 3 -4-5-6-7-8.

Nessa perspectiva o presente trabalho visa abordar a relação existente entre o HPV e o carcinoma epidermoide bucal (CEB). Justifica-se sua realização pela medida em que este reúne informações importantes acerca de uma doença infecciosa que afeta milhares de indivíduos no mundo, apontando, inclusive, sua relação com o CEB. Para tal, realizou-se uma revisão de estudos disponíveis em bases de dados on-line durante o período de 2014 a 2019, analisando-os de maneira qualitativa.

\section{METODOLOGIA}

Para o desenvolvimento deste estudo de revisão, realizou-se um levantamento da literatura publicada entre 2014 e 2019, a respeito da relação entre o Papilomavírus Humano e o CEB, por meio de busca on-line nas bases de dados Portal de Periódicos CAPES, Scientific Library Online (SciELO), Medline-Pubmed, Revista Eletrônica de Odontologia (RevOdonto) e Biblioteca Virtual em Saúde (BVS/ LILACS). As palavras-chave utilizadas na busca foram Câncer Bucal, Carcinoma Epidermoide, HPV-16, HPV-18, Papilomavírus Humano e suas respectivas traduções para a língua inglesa.

Os critérios de seleção para inclusão foram (1) publicação entre 2014 e 2019, (2) abordagem sobre a relação do HPV e o carcinoma epidermoide bucal, (3) pesquisas realizadas com público adolescente, adulto e idoso e (4) estudos em português, inglês e espanhol.

Os critérios para exclusão foram (1) publicações anteriores a 2014, (2) utilização de animais como meio de estudo, (3) pesquisas realizadas com público infantil (de 0 a 12 anos incompletos) e (4) estudos em línguas diferentes de português, inglês e espanhol. 


\section{REVISÃO DE LITERATURA}

\subsection{Características virais}

As partículas virais do Papilomavírus Humano estão, taxonomicamente, classificadas na família Papillomaviridae e no gênero Alphapapillomavirus. Esses vírus não são envelopados, possuem tamanho nanométrico e apresentam genoma circular de fita dupla do tipo ácido desoxirribonucleico (DNA $)^{6-7}$.

A infecção pelo HPV inicia-se quando as partículas virais, por meio de uma microabrasão nos tecidos e mucosas, adentram as células basais e se tornam progressivamente diferenciadas ${ }^{7-14}$.

Por meio dos genes precoces E6 e E7, que possuem potencial oncogênico, o vírus realiza a degradação da proteína 53 (p53) e inibição da proteína retinoblastoma $(\mathrm{Rb})$, o que acarreta em supressão da realimentação do ciclo celular ${ }^{7,9}$. Além disso, é possível evidenciar um processo de metilação que provoca mutação e exclusão da proteína 16 (p16), ocasionando alteração do ciclo celular normal ${ }^{9-10-11}$.

A infecção pelo HPV ocorre de maneira mais eficiente nos queratinócitos basais do epitélio da cripta reticular situados nos tecidos linfoepiteliais, ocasionando uma infecção persistente, com expressão oncoproteica estável e induzindo à carcinogênese celular ${ }^{11}$. Além do exposto, destaca-se que o HPV é capaz de contribuir para a transformação maligna de uma célula por meio da desregulação do ciclo celular $^{7-9-12}$, inibição da apoptose e indução de instabilidade genética e angiogênese ${ }^{13}$.

No diagrama-resumo, a seguir, apresenta-se o ciclo carcinogênico do vírus HPV nas células infectadas.

Figura 1 - (A) Diagrama demonstrando a ligação da proteína ao fator de transcrição E2F resultando na interrupção do ciclo celular. A fosforilação da pRb libera E2F que ativa a transcrição de genes alvo para replicação do genoma. A proteína viral $E 7$ se liga a proteína $p R b$, isso libera $E 2 F$, causando um estímulo constante para a divisão celular. (B) A proteína viral E6 se liga à E6AP que funciona como uma ubiquitina, degradando p53 e permitindo rearranjos mutacionais.

(A)

\section{TRANSFORMAÇÃO TUMORAL}

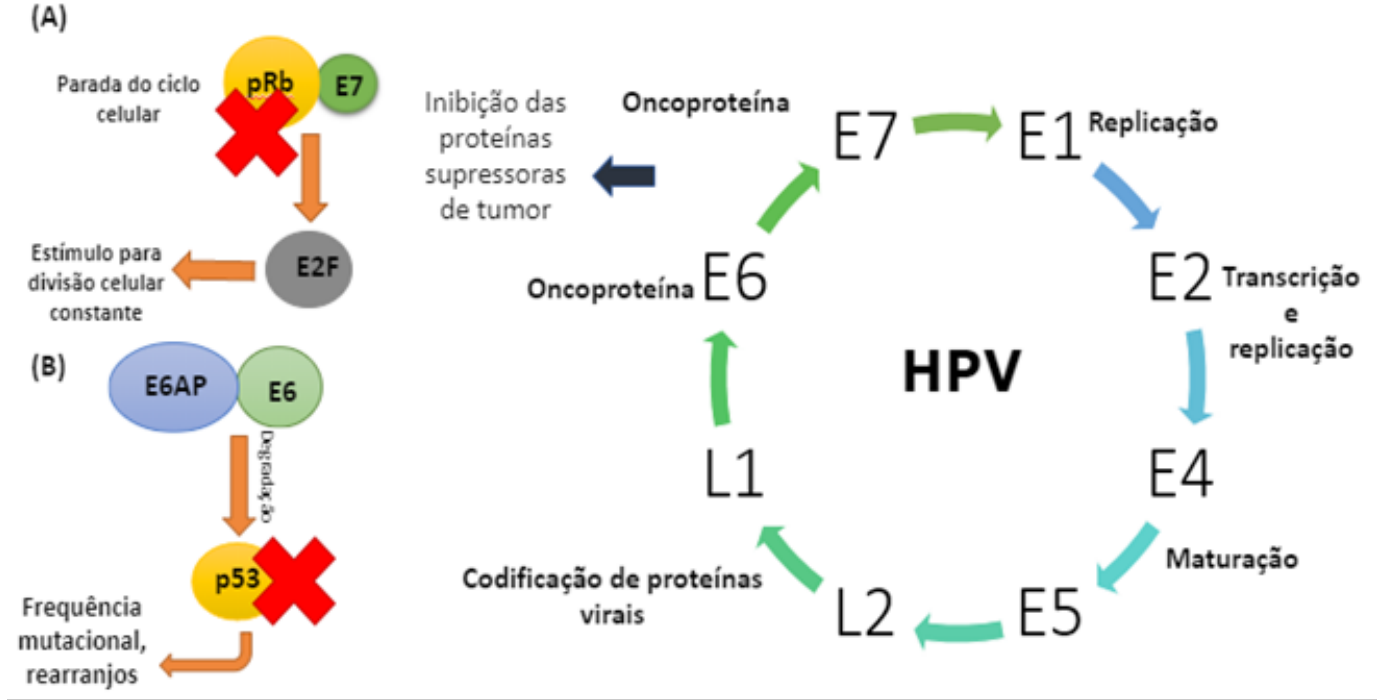

Fonte: Acervo dos autores, 2019. 


\subsection{Relação entre o HPV e o carcinoma epidermoide bucal}

Uma grande variedade de estudos afirma que o HPV pode ser relacionado ao CEB pelo fato de o vírus apresentar tropismo pelas células da membrana de revestimento da cavidade bucal, que apresentam características histopatológicas semelhantes com as células do colo do útero (sítio comprovadamente relacionado como passível de infecção pelo HPV) ${ }^{11-13-14-15-16 . ~ A d e m a i s, ~ e ́ ~ d e s c r i t o ~ q u e ~ o ~ H P V ~}$ pode ocasionar, sobretudo, alterações na orofaringe ${ }^{16-17}$.

Na literatura utilizada para confecção do presente trabalho, é possível notar a presença de estudos clínicos que evidenciam a correlação positiva entre o CEB e o HPV. Dentre eles, cita-se uma pesquisa realizada com 173 indivíduos portadores do carcinoma epidermoide bucal, em que foi demonstrado que 18,5\% participantes apresentavam também infecção pelo vírus HPV e, por meio de biópsia das lesões, foi possível notar a predominância do vírus HPV-16 nas células analisadas?

Resultados semelhantes foram encontrados por Candotto et al. ${ }^{5}$, em que a análise de amostras celulares de 23 portadores de CEB detectou a presença do vírus HPV-16 em uma gama considerável das amostras coletadas. Entretanto, outras análises e estudos demonstram que o subtipo viral de HPV mais associado ao CEB é o HPV-18 ${ }^{11}$.

\subsection{Epidemiologia}

A prevalência de HPV mostra-se variável em diversos estudos e em diferentes regiões geográficas. Infecções da cavidade bucal por HPV são maiores entre os indivíduos do sexo masculino, de meia-idade e com baixas condições socioeconômicas ${ }^{18-11-19}$, enfatizando-se que tal prevalência varia amplamente de acordo com fatores demográficos, influenciados pelas diferenças culturais ${ }^{11-19}$.

Notou-se também, durante a leitura dos estudos que compõem o presente trabalho, que o padrão de vida predominante na sociedade contribui para que o indivíduo contemporâneo esteja se sujeitando a hábitos e práticas nocivas para o seu bem-estar físico e psíquico, aumentando cada vez mais o risco de adquirir doenças, principalmente as sexualmente transmissíveis ${ }^{11-13-20-21}$.

Estudos relatam uma forte associação entre o HPV e as lesões bucais malignas, principalmente em indivíduos jovens (até 45 anos) que não fumam e não bebem em excesso, mas que praticam sexo oral e sexo desprotegido ${ }^{11-13-22-23}$. Destaca-se que, no Brasil, a taxa de mortalidade, no ano de 2013, por tal infecção foi de 5.401 casos, sendo 4.223 homens e 1.178 mulheres $^{11}$.

\subsection{Fatores de risco}

O carcinoma epidermoide é uma patologia multifatorial que apresenta como fatores etiológicos as condições culturais, a prática sexual desprotegida ou com múltiplos parceiros, o alcoolismo, etilismo, as más condições de higiene bucal e as características hereditárias ${ }^{4-7-21-22-24-25-26-27-28}$.

Estudos apontam que o Papilomavírus Humano intensifica um crescimento celular afinalístico, agindo

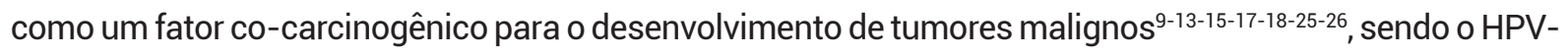
16 e o HPV-18 os subtipos de Papilomavírus que possuem maior capacidade de ocasionar o CEB ${ }^{4-9-13-18}$. 


\subsection{Sítios bucais mais afetados pelo CEB}

0 câncer de boca caracteriza-se como um grupo de lesões malignas que provocam o crescimento afinalístico e acelerado das células encontradas nos mais diversos tecidos bucais ${ }^{22}$. Análises clínicas e histopatológicas permitiram constatar que, dentre os sítios bucais mais afetados pelo CEB, estão o assoalho bucal, a língua, o palato, as tonsilas palatinas e a mucosa jugal 20-22-27-28-29-30-31.

No estudo epidemiológico-clínico de Mushfiq et al. ${ }^{32}$ foi enfatizado que os tumores associados ao HPV-16 e HPV-18 localizados na língua e na área gengival podem ocasionar consequências mais graves para os pacientes, como, por exemplo, incapacidade de deglutir, mastigar e dificuldades durante a emissão da fala, sendo que elevados índices de mortalidade são associados aos cânceres epidermoides bucais que se desenvolvem na região lingual ${ }^{28}$.

\subsection{Transmissão}

Para que o vírus infecte um indivíduo, é necessário que haja pequenas lesões nos tecidos que facilitem o contato de tal entidade com as células basais. Sua transmissão pode ocorrer verticalmente, por meio da transmissão perinatal de origem cervical e, horizontalmente, por meio da prática sexual desprotegida ${ }^{15-23-30}$.

\subsection{Sinais e sintomas do CEB}

Em alguns indivíduos acometidos pelo CEB, é possível verificar a presença de lesões de difícil cicatrização, disfagia, manchas brancas e vermelhas, úlceras, massas tumorais, hipermobilidade dental, trismo e parestesia ${ }^{22-23-30-33}$. De acordo com o INCA' ${ }^{1}$, comumente é possível notar também a presença de odinofagia, fadiga, falta de apetite, náuseas, vômitos, edema, linfoedema, sangramento, desconforto em orofaringe e dificuldade para movimentar a língua.

\subsection{Diagnóstico e Tratamento}

O processo de diagnóstico do carcinoma epidermoide bucal é realizado, principalmente, por meio de biópsia incisional e raspagem da região acometida para posterior análise laboratorial1-3-31. Destaca-se que o exame clínico associado à biópsia com o estudo da lesão por tomografia permite ao profissional definir um diagnóstico precoce aliado a um tratamento adequado, proporcionando ao paciente um melhor prognóstico'.

O CEB deve ser tratado por meio da excisão cirúrgica da lesão, quimioterapia e/ou radioterapia. Entretanto, a realização de quimioterapia e radioterapia ocasiona graves efeitos adversos para os pacientes por possuírem elevadas taxas de toxicidade ${ }^{29}$. No entanto, alguns estudos indicam que pacientes acometidos concomitantemente por HPV e CEB apresentam melhores prognósticos, visto que esse vírus aumenta a sensibilidade do tumor ao tratamento radioterápico e quimioterápico, o que permite a redução da quantidade de sessões de tais tratamentos e, por conseguinte, proporciona uma maior sobrevida ao paciente $\mathrm{e}^{15-23}$.

Em um estudo no qual foi testada a sensibilidade de células bucais acometidas por CEB e pelo vírus HPV foi possível notar um aumento significativo da sensibilidade intrínseca dessas células aos tratamentos radioterápicos e quimioterápicos, quando comparadas àquelas que apresentavam apenas o CEB ${ }^{23}$. Esse mecanismo associado a uma melhor resposta nos cânceres bucais não é claro e precisa de mais investigação, entretanto a redução nas doses de quimioterapia e radioterapia demonstra-se como uma vantagem evidente da infecção por HPV em indivíduos acometidos pelo CEB ${ }^{23}$. 


\subsection{Vacina contra o HPV}

Uma das estratégias utilizadas para reduzir a contaminação pelo HPV é a vacinação, importante fator profilático contra o vírus ${ }^{29}$. Kim et al. ${ }^{15}$ mostraram que indivíduos vacinados contra o microrganismo apresenta menor prevalência da doença, o que indica sua eficácia.

Nesse sentido, ressalta-se que a característica básica de uma vacina é recrutar células para atuar na imunização contra determinado fator patogênico. As vacinas quadrivalentes contra o Papilomavírus Humano, encontradas na rede particular, possuem uma ampla imunização que evita a infecção pelos subtipos 9, 11, 16 e 18. Já a vacina bivalente, demonstra-se eficiente contra o HPV dos tipos 16 e $18^{35}$.

\subsection{A importância da atuação do cirurgião-dentista}

Agir em prol da ação do cuidar, atuando nas manifestações de patologias, na promoção da qualidade de vida e na intervenção em fatores que colocam a saúde dos indivíduos em risco é papel do cirurgião-dentista ${ }^{35}$. Entretanto, barreiras sociais ainda precisam ser ultrapassadas para que esses profissionais consigam intervir de maneira eficaz em fatores determinantes para o processo de saúde-doença de seus pacientes ${ }^{33-35}$. Tais barreiras incluem, principalmente, a dificuldade da abordagem de assuntos relacionados a doenças sexualmente transmissíveis, como o HPV ${ }^{17-33}$.

Ressalta-se, ainda, que o exame clínico e o adequado acompanhamento permitem que ocorra a detecção de diversas lesões, anomalias e alterações na cavidade bucal ${ }^{22-35}$. Ademais, a identificação precoce de lesões potencialmente malignas, como o carcinoma epidermoide bucal, permite que as chances de cura aumentem consideravelmente ${ }^{33}$.

Apesar da importância dos cirurgiões-dentistas nesse processo que envolve o HPV e o carcinoma epidermoide, tais profissionais não sabem a forma correta de atuar em tal questão $0^{17-33-36-37}$.

Segundo uma pesquisa realizada por Poelman et al..$^{37}$, na Universidade de Odontologia da Jordânia, mais da metade dos estudantes entrevistados apresentavam baixo nível de capacitação para realizar a triagem de um câncer de boca e de uma infecção por HPV. Tal dado evidencia a necessidade de uma maior abordagem durante o curso de formação dos graduandos em Odontologia sobre as doenças infecciosas e suas consequências para a manutenção da homeostase corporal ${ }^{37}$. Portanto, a atuação do cirurgião-dentista deve ir além da realização de estudos, procedimentos e tratamentos odontológicos de rotina. É dever desses profissionais atuarem na promoção de saúde, disseminação de conhecimentos

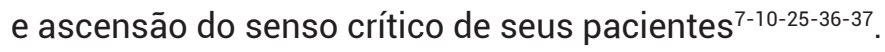

\section{CONCLUSÃO}

Devido ao novo padrão de vida predominante na sociedade, o indivíduo contemporâneo está sujeitando-se de forma exacerbada a hábitos e práticas nocivas para o seu bem-estar físico e psíquico, aumentando o risco de adquirir doenças sexualmente transmissíveis, como, por exemplo, o HPV. A literatura pesquisada aponta uma correlação do HPV com o CEB devido ao fato de o vírus possuir tropismo pelas células da membrana de revestimento da cavidade bucal e apresentar características histopatológicas semelhantes às células do colo do útero. Apesar disso, se faz necessária a realização de mais estudos para estabelecer uma relação concreta. Ademais, visto a despreparação da maioria dos cirurgiões-dentistas na atuação do diagnóstico do câncer de boca e de infecção por HPV, pode-se sugerir que é necessário haver uma mudança na conduta de realização de exames clínicos e tratamentos odontológicos, uma vez que, em sua maior parte, são realizados com a intenção de tratar sintomas e não causas, interferindo diretamente nos prognósticos de patologias. 
Amanda Andressa de Souza Carvalho / Graziele de Souza Diniz / Lúbia Cerqueira Costa Stéphany Souza de Araújo / Prof. Dr. Josemar Parreira Guimarães

\section{REFERÊNCIAS}

1. INCA. Câncer de Boca. Instituto Nacional do Câncer (Brasil). Tipos de Câncer. Câncer de Boca, 2019. Disponível em: https://www.inca.gov.br/tipos-de-cancer/cancer-de-boca. Acesso em: 10 abr. 2019.

2. KUNDU, S. et al. Association of DFNA5, SYK, and NELL1 variants along with HPV infection in oral cancer among the prolonged tobacco-chewers. Tumor Biology, v. 40, n. 8, 2018.

3. SILVA, F. J. Influência do HPV como potencial fator de risco de desenvolvimento do carcinoma de células escamosas intraoral: uma revisão de literatura. Trabalho de Conclusão de Curso (Monografia) - Odontologia, Universidade Federal de Sergipe- UFS, Lagarto, 2018.

4. URSU, R. G. et al. Role of mucosal high-risk human papillomavirus types in head and neck cancers in Romania. Plos One, v. 13, n. 6, 2018.

5. CANDOTTO, V. et al. HPV infection in the oral cavity: epidemiology, clinical manifestations and relationship with oral cancer. Oral Implantol, v. 10, n. 3, p. 209, 2017.

6. SYRJÄNEN, S. Oral manifestations of human papillomavirus infections. Eur J Oral Sci, v. 126, n. 1, p. 49-66, 2018.

7. MAIA, L. M. S. et al. Correlação das infecções causadas pelo vírus do papiloma humano com os casos de câncer de boca e orofaringe no Mato Grosso. Trabalho de Conclusão de Curso (Monografia) -Biomedicina, Universidade Federal do Mato Grosso- UFMT, Cuiabá, 2016.

8. GUO, L. et al. Prevalence of human papillomavirus type-16 in head and neck cancer among the Chinese population: a meta-analysis. Front Oncol, v. 8, p. 619, 2018.

9. KOUKETSU, A. et al. Detection of human papillomavirus infection in oral squamous cell carcinoma: a cohort study of Japanese patients. J Oral Pathol Med, v. 45, n. 8, p. 565-572, 2016.

10. LIU, T. et al. Study on expression of p16 and human papillomavirus 16 and 18 (E6) in OLP and its malignant transformation. Pathol Res Pract, v. 214, n. 2, p. 296-302, 2018.

11. GUO, L. et al. Prevalence of human papillomavirus type-16 in head and neck cancer among the Chinese population: a meta-analysis. Front Oncol, v. 8, n. 3, p. 619, 2018.

12. BARRETO, R. C. et al. Relação papilomavírus (HPV) e tumor maligno da cavidade bucal. Rev Bras Ciênc Saúde, v. 18, n. 3, p. 261-70, 2014.

13. MONTENEGRO, L. A. S. et al. Papiloma vírus humano como fator carcinogênico e co-carcinogenico do câncer oral e da orofaringe. ROBRAC, v. 23, n. 67, 2014.

14. POELMAN, M. R. et al. Prevention of HPV-related oral cancer by dentists: assessing the opinion of Dutch dental students. J Cancer Educ, v. 33, n. 6, p. 1347-1354, 2018.

15. KIM, S. M. et al. Identification of human papillomavirus (HPV) subtype in oral cancer patients through microarray technology. Eur Arch Oto-Rhino-L, v. 275, n. 2, p. 535-543, 2018. 
16. DOORBAR, J. et al. Human papilomavírus molecular biology and disease association. Rev Med Virol, v. 25, p. 2-23, 2015.

17. MORAIS, E. F. et al. Avaliação do efeito carcinogênico do papilomavírus humano em cavidade oral e orofaringe: Uma revisão sistemática. Rev Méd Minas Gerais, v. 26, 2017.

18. OWOSHO, A. A. et al. Epidemiological trends of oropharyngeal and oral cavity squamous cell carcinomas in Northern New England, 2000-2013. Cancer Cause Control, v. 30, n. 3, p. 291-299, 2019.

19. KHYANI, I. A. M. et al. Salivary detection of human Papilloma virus $16 \& 18$ in pre-malignant and malignant lesions of oral cavity: Is it feasible in Pakistani context of Socio-Cultural Taboos?. Pak J Med Sci, v. 31, n. 5, p. 1104, 2015.

20. BANDHARY, S. K. et al. Detection of Human Papilloma Virus and Risk Factors among Patients with Head and Neck Squamous Cell Carcinoma Attending a Tertiary Referral Centre in South India. Asian Pac J Cancer P, v. 19, n. 5, p. 1325, 2018.

21. CAMACHO AGUILAR, S. et al. Human papillomavirus load in benign HPV associated oral lesions from HIV/AIDS individuals. Oral Dis, v. 24, n. 1-2, p. 210-214, 2018.

22. FERREIRA, L. L. Comparação da prevalência do HPV- 16 em pacientes com carcinoma espinocelular de boca e lesões potencialmente malignas bucais. Tese (Doutorado em Odontologia). UNESP, Universidade Estadual Paulista, Câmpus de Araçatuba, Araçatuba, 2017.

23. GREWAL, R. K. et al. Detection of human papilloma virus-E6/E7 proteins of high-risk human papilloma virus in saliva and lesional tissue of oral squamous cell carcinoma patients using nested multiplex polymerase chain reaction: A comparative study. J Oral Maxillofac Pathol, v. 22, n. 3, p. 318, 2018.

24. SALLAM, M. et al. Dental students' awareness and attitudes toward HPV-related oral cancer: a cross sectional study at the University of Jordan. BMC Oral Health, v. 19, n. 1, p. 171, 2019.

25. KHOT, K. P.; DESHMANE, S.; CHOUDHARI, S. Human papilloma virus in oral squamous cell carcinomathe enigma unravelled. Chin J Dent Res, v. 19, n. 1, p. 17-23, 2016.

26. QATOUSEH, L. A. et al. Detection of High-Risk Human Papillomavirus Genotypes 16 and 18 in Head and Neck Squamous Cell Carcinomas in Jordan. Asian Pac J Cancer P, v. 18, n. 5, p. 1337, 2017.

27. ASHRAF, M. J. et al. The prevalence of human papilloma virus in squamous cell carcinoma of oral tongue. Iranian J Pathol, v. 12, n. 2, p. 144, 2017.

28. VEITÍA, D. et al. Prevalence of HPV and EBV infection and their relationship with the p53 and PCNA expression in oral carcinoma patients. J Oral Res, v. 6, n. 4, p. 86-91, 2019.

29. YETE, S.; D'SOUZA, W.; SARANATH, D. High-risk human papillomavirus in oral cancer: clinical implications. Oncology, v. 94, n. 3, p. 133-141, 2018.

30. PANNEERSELVAM, K. et al. Detection of human papillomavirus 16 and 18 in patients with oral squamous cell carcinoma and potentially malignant oral disorders in South Indian population: A pilot study. J Cancer Res Ther, v. 15, n. 3, p. 571, 2019. 
31. MARQUES, M. P. C. et al. Comparative study between biopsy and brushing sampling methods for detection of human papillomavirus in oral and oropharyngeal cavity lesions. Braz J Otorhinol, v. 81, n. 6, p. 598-603, 2015.

32. SHAIKH, M. H. et al. cGAS-STING responses are dampened in high-risk HPV type 16 positive head and neck squamous cell carcinoma cells. Microb Pathog, v. 132, p. 162-165, 2019.

33. SOARES, A. C. R.; PEREIRA, C. M. Associação do HPV e o Câncer Bucal. Rev Ciênc Odonto, v. 2, n. 2, p. 22-27, 2018.

34. BRASIL, Ministério da saúde. Secretaria de atenção a saúde. Departamento de atenção básica, coordenação nacional de saúde bucal: Diretrizes da política nacional de saúde bucal. Brasília (DF), 2004. Disponível em: https://bvsms.saude.gov.br/bvs/publicacoes/politica_nacional_brasil_ sorridente.htm. Acesso em: 27 abr. 2019.

35. Instituto Nacional de Câncer José Alencar Gomes da Silva (Brasil). Coordenação de Prevenção e Vigilância, Divisão de Deteç̧ão Precoce e Apoio à Organização de Rede: Diretrizes brasileiras para o rastreamento do câncer do colo do útero. Rio de Janeiro. Disponível em: https://www.inca.gov. $\mathrm{br} /$ publicacoes/livros/diretrizes-brasileiras-para-o-rastreamento-do-cancer-do-colo-do-utero. Acesso em: 27 abr. 2019.

36. SIMOES, L. P.; JUNIOR, G. Z. Vírus HPV e o desenvolvimento de câncer de colo de útero-uma revisão bibliográfica. Rev Uningá, v. 56, n. 1, p. 98-107, 2019.

37. POELMAN, M. R. et al. Prevention of HPV-related oral cancer by dentists: assessing the opinion of Dutch dental students. J Cancer Educ, v. 33, n. 6, p. 1347-1354, 2018. 